\title{
Identification of focus areas for Australian Bat Lyssavirus potential exposure prevention in the Metro North Hospital and Health Service region
}

Short title: Spatiotemporal analyses of ABLV exposure

Fiona J May ${ }^{1}$, Kay D Mann ${ }^{1,2}$, Daniel P Francis ${ }^{1}$, Megan K Young ${ }^{1,3}$

${ }^{1}$ Metro North Public Health Unit, Queensland Health, Windsor, Brisbane, Australia

${ }^{3}$ Griffith University School of Medicine and Menzies Health Institute Queensland, Southport, Gold

Coast, Australia

Current affiliation:

${ }^{2}$ Commonwealth Scientific and Industrial Research Organisation, Herston, Brisbane, Australia

\section{ORCID}

Fiona J May https://orcid.org/0000-0003-1306-0179

Kay D Mann https://orcid.org/0000-0003-0115-5293

Daniel P Francis https://orcid.org/0000-0002-1031-9969

Megan K Young http://orcid.org/0000-0003-1241-3366

\section{Acknowledgments}

With thanks to staff at the Metro North Public Health Unit for their assistance navigating the filing system. 


\section{Summary}

Australian Bat Lyssavirus (ABLV) is a fatal rabies-like disease spread to humans from bats. All people who report bat bites or scratches in Queensland are considered potentially exposed to ABLV and are followed up and treated to prevent ABLV. Preventing members of the public intentionally interacting with bats will reduce the number of potential exposures to ABLV. In order to target public health messaging, this study examines the epidemiology of potential ABLV exposures in Metro North Hospital and Health Service (HHS), a region of Queensland that encompasses metro and rural areas in the south east of the state.

People who intentionally handled bats during the study period were more likely to be adult (93\%), male $(60 \%)$, scratched $(51 \%)$ by a megabat $(72 \%)$, and been potentially exposed while rescuing the bat when it was trapped or injured (72\%). The number of potential exposures reported in Queensland has increased since 2013, the same year a Queensland child died of ABLV. Seasonally, exposures are more common during bat breeding and nursing periods when bats are more active (summer to autumn). Although there were more notifications in a band stretching north from the inner city to northern metro suburbs, notification rates were higher in large rural statistical areas in the north of the HHS. These data will be used to develop geographically targeted ABLV prevention messaging for the general public.

Key words: Australian bat lyssavirus, spatio-temporal analysis, public health, SaTScan

\section{Impacts}

- Australian bat lyssavirus is a fatal disease spread to humans through contact with bats, but many people intentionally contact bats, putting themselves at risk of infection

- Response to potential exposure to Australian bat lyssavirus (high risk contact with a bat) is expensive and time consuming for public health and unpleasant for those exposed

- This study uses spatio-temporal techniques to find focus areas where strategies to educate people about safe bat contact can be targeted to those most likely to benefit 


\section{Introduction}

Australian bat lyssavirus (ABLV) is in the Lyssavirus genus and is related to rabies virus. It occurs naturally in the Australian bat population and may be transmitted from bats to humans via bites, scratches, or mucous membrane or broken skin exposure to bat saliva (Australian Government Department of Health, 2013). ABLV causes a rabies-like illness in humans and has to date caused three fatalities in Australia, all of them in Queensland (Francis et al., 2014; Hanna et al., 2000; Murray, Morgan, \& Allworth, 1996).

Testing a bat for ABLV requires euthanasia of the bat. As a result, testing of bats is biased towards those more likely to be infected, resulting in an overestimate of the prevalence of ABLV. However, it has been estimated that less than $1 \%$ of healthy, wild flying foxes (a type of large bat) and insectivorous bats and $5-10 \%$ of sick, injured or orphaned bats in Queensland, Australia, are infected with ABLV (Field, 2004; McCall et al., 2000; Merritt et al., 2018; Warrilow et al., 2003). A recent publication reported that in 2017 in Australia, 28 bats (6.7\% of all those tested) were found to have ABLV infection, and 19 of these were bats found in Queensland (Wildlife Health Australia, 2017).

Under the Queensland Public Health Act 2005 any potential ABLV exposure is a notifiable event (Queensland Health, 2005). This requires notification to a local public health unit of any bite, scratch, or mucous membrane or broken skin exposure to the saliva or neural tissues of a bat in Australia or overseas. These strict notification guidelines enable the timely administration of post-exposure prophylaxis to protect the individual as without early treatment before development of clinical disease ABLV infection in humans is fatal. Following notification, the public health unit interviews the person who was potentially exposed to obtain further information about the bat encounter and to determine if prophylaxis is required. This information is forwarded to the Queensland Notifiable Conditions System (NoCS) as required under the Act.

Post-exposure prophylaxis includes a course of rabies vaccines and normally rabies immunoglobulin. Prophylaxis may not be required if the bat involved is available for testing and returns a negative result for lyssavirus. However, if the bat is not available or cannot be tested, post-exposure prophylaxis is recommended as a precaution. While post-exposure prophylaxis is safe, there are known side-effects related to administering these products, and they are costly to the health service. At approximately AU\$300 for a vial of immunoglobulin and AU\$70-120 per vaccine, the cost of a course of post-exposure prophylaxis is around $\mathrm{AU} \$ 1800$ per person in addition to staffing costs. 
Considering the costs to the health service and the inconvenience and potential adverse events to recipients, it is desirable to prevent as many potential exposures as possible.

Previous studies on potential ABLV exposures have reported a reduction in potential ABLV exposure notifications in South East Queensland between 1996 and 2008, but highlight that over half of the notifications recorded were the result of intentional handling of bats by members of the public (M. K. Young \& McCall, 2004; Megan K. Young \& McCall, 2010). This equates to hundreds of preventable potential ABLV exposures occurring in Queensland every year. Current public health messaging, advising people not to handle bats, but to contact a vaccinated trained handler to assist, is released to the media by the Department of Health each year and is readily available on the Queensland Health website. These state-wide approaches to prevention do not seem to have resulted in a decrease in preventable potential exposures (Megan K. Young \& McCall, 2010).

It may be that strategies to raise awareness of ABLV would benefit from considering both the content of the messaging and whether it should be targeted at specific population(s) at greater risk of handling bats. Recent research suggests that messaging should emphasise the risk that handling of bats by unvaccinated people poses to themselves as well as to the bat (M. K. Young, El Saadi, \& McCall, 2014). Additionally, in circumstances when avoidance is not considered to be possible, alternative options to touching bats with hands should be communicated (such as when people deem their pet or child to be in danger) (M. K. Young et al., 2018).

Where and how this message should be targeted is less clear and is complicated by the widespread nature of bat activity. Nonetheless, understanding the epidemiology and distribution of bat exposures would provide helpful intelligence to inform public health activity. The aim of this study was to examine the epidemiology of preventable potential ABLV exposures in the Metro North Hospital and Health Service (HHS) region of south east Queensland (our local public health unit jurisdiction) to identify areas that might benefit from geographical targeting of prevention messages. Particularly, we aimed to identify if preventable potential exposures were clustered geographically within our jurisdiction.

\section{Methods}

\section{Exposure data}

All human potential ABLV exposures in residents of the Metro North HHS that were notified between 1 January 2007 to 31 December 2017 were identified from an audit of Metro North Public Health Unit case report forms (CRF). The following data were extracted for each potential exposure: 
unique notification identifier, notification date, potential exposure date, sex of the individual, age at potential exposure, residential address, the circumstances and location of the potential exposure, bat type, potential exposure type, whether the potential exposure was preventable and whether the individual was a bat carer.

Potential exposures were categorised as reported in Young et al (2010) and only those in the category "General public, intentional bat handling" were included in the analysis. This category includes people who attempted to rescue or free a bat from netting or fencing, or picked up a bat found lying on the ground. Unintentional potential exposures, potential exposures of bat carers and their families, and potential exposures of those who handled bats as part of their profession were excluded.

Further categorisation of preventable potential exposures was based on information provided in the CRF. Free text descriptions of the nature of the bat contact were allocated to one of four groups: rescued a trapped or injured bat (such as removing a bat from fruit tree netting or picking up a sick or injured bat from the ground), removed a bat from an animal or person (such as rescuing a bat from the family dog, or removing a bat that was attached to a person), removed a bat from the home, and "other".

Each case was categorised as adult (18 years and older) or child (under 18 years) using the age at exposure recorded on the CRF. The residential address of each case was substituted when the location of bat exposure was not available.

Population data

Estimated resident population (ERP) data by Statistical Area Level 2 (SA2) for the Metro North HHS region were obtained from the Queensland Health InfoBank. These data are adapted by the Queensland Health Statistical Services Branch from the Australian Bureau of Statistics (ABS) data to account for HHS boundaries as some HHS boundaries cross through SA2s (e.g. Kilcoy SA2). Preventable potential ABLV exposure incidence rates per SA2 were calculated using the 2012 ERP. SA1 ERPs were calculated by combining meshblock (the smallest ABS geography) populations available from the ABS as some SA1s are also bisected by HHS boundaries. Meshblock ERPs were available for 2011 to 2017.

GIS

Addresses were geo-coded using Public Sector Mapping Agencies (PSMA) Australia's Geocoded National Address File (G-NAF) dataset (G-NAF CPSMA Australia Limited licensed by the Commonwealth of Australia under the Open Geo-coded National Address File (G-NAF) End User 
Licence Agreement., 2018) and plotted using Quantum Geographical Information Software (QGISTM (QGIS Development Team, 2018)) . Where only the suburb of exposure was available, the centroid of the suburb was used for geocoding.

Shapefiles for SA1, SA2 and SA3 areas were downloaded from the ABS. HHS shapefiles were obtained from Queensland Health. Where required, individual SA1, SA2 and SA3 features were reshaped to match HHS borders.

Individual SA2s and SA1s are designed to have approximately the same population across Australia (3 000 - 25000 people [average 10000 ] and $200-800$ people [average 400 ] respectively). As a result, the areas of SA2S with high population densities are much smaller than those in areas with low population density. The size of an area determined to be feasible for a geographic public health prevention focus was determined by consensus to be 0.05 degrees. A grid of squares corresponding to this area was laid over the Metro North shapefile. Using map projection GDA94 for the Metro North area, the resulting squares had an area of $27.5 \mathrm{~km}^{2}$. The size of the grid was chosen to produce areas that were practical for targeting of public health messaging.

\section{Statistical analysis}

Descriptive statistics of the number of preventable potential ABLV exposures in the Metro North HHS region were calculated by age, sex, potential exposure type, bat type (microbat or megabat), category of contact and potential exposure time. Total numbers of preventable potential ABLV exposures were aggregated by month and year, using potential exposure date, and plotted to illustrate seasonal variation.

Using the borders of the uniform grid laid over the Metro North shapefile, the number of preventable potential exposures within uniform areas of $27.5 \mathrm{~km}^{2}$ were counted.

Rates of preventable potential exposures within SA2 areas and counts within SA3 areas were mapped across the Metro North HHS region.

Geographic clusters of preventable potential ABLV exposures were determined using SaTScan ${ }^{\mathrm{TM}}$ software, version 9.6, using the Kulldorff method of retrospective Poisson space-time modelling (Kulldorff, 1997). This method scans a cylindrical window of varying sizes across space and time noting the number of observed and expected ABLV potential exposures inside the window at each location. Total numbers of preventable potential ABLV exposures per SA1 per year along with ERPs per SA1 per year (2011 - 2017 ERPs were used to impute 2007 - 2010 ERPs) were used. The expected number of potential exposures were calculated under the null hypothesis that they were 
independent (i.e. no clusters). A discrete Poisson model was used to identify clusters with a higher than expected number of potential exposures, allowing adjustment for the uneven geographic density of background population. Both spatial only and spatio-temporal models were tested. The maximum spatial cluster size was set to $10 \%$ of the population at risk and for the spatio-temporal model, the time precision was set to one year and the temporal window set to $90 \%$ of the study period (for space-time analysis). Spatial only and temporal only clusters were allowed in the spatiotemporal analysis to detect clusters of higher than expected cases that spanned the whole of Metro North HHS or the whole period 2007 to 2017 respectively and the temporal window of $90 \%$ of the study period will detect all clusters spanning less than the whole period. Likelihood ratio tests using 9999 Monte Carlo replications were used to detect clusters at a significance level of $p<0.05$.

Ethics

As a service quality assurance and evaluation activity, this study was granted exemption from human research ethics committee review by the Royal Brisbane and Women's Hospital Human Research Ethics Committee chairperson on 28/6/2018, reference HREC/18/QRBW/362.

\section{Results}

\section{Descriptive}

Between January 2007 and December 2017 there were 148 preventable potential ABLV exposures in residents of the Metro North HHS region. Four exposures where the exposure location was known to be at a location other than their residence but the location could not be identified were excluded. After exclusions, a total of 144 preventable potential ABLV exposures were included in the sample for analysis.

Of the 144 exposures, $48(33 \%)$ were exposed at their residential address, $14(10 \%)$ were exposed outside the home at a known location, $1(1 \%)$ was exposed outside the home but only a suburb of exposure was known and for 81 exposures (56\%) the exact location of exposure was unknown but assumed to be near their home (e.g. people who were out walking when they encountered the bat or rescuing a bat from netting without specifying whether it was on their own property). For this final category, the residential address was used as the location of exposure.

The people reporting preventable potential ABLV exposures ranged in age from 5 to 85 years old (median 49 years old) and were mainly in adults (93\%). Most potential exposures were a scratch (51\%), in males (60\%), involving megabats $(72 \%)$ and were evenly split between morning and 
evening (Table 1). Most people received their potential ABLV exposure while rescuing a trapped or injured bat (72\%).

Between 1 January 2007 and 31 December 2017, total numbers of preventable potential ABLV exposures ranged from 5 to 29 per year with a mean of 13 per year. Between 2013 and 2017 (mean 19 exposures per year) there was a sustained increase in the number of exposures recorded compared to the preceding 5-year period (mean 8 exposures per year, $2007-2012$; Figure 1). The incidence of preventable potential ABLV exposures for the 11 years of study (2007 - 2017) was 16 per 100000 people, an average of 1.4 exposures per 100,000 population per year.

\section{Seasonality}

There was on average one preventable potential ABLV exposure per month (range 0 - 5) with peaks in October - November and January - February. There was a drop in preventable potential ABLV exposures in December (Figure 2).

\section{Preventable potential exposure counts in squares of uniform land area}

Using equal sized grid squares over the Metro North HHS region identified some areas that had higher numbers of exposures regardless of the underlying population (Figure 3). These included SA2s from north of the inner city through a corridor up to Bracken Ridge, with a peak of exposures through Stafford, Chermside and Aspley. The Deception Bay area also had a higher number of exposures than surrounding areas.

\section{Preventable potential exposure Incidence rates by SA2 and counts by SA3}

The rates of preventable potential ABLV exposures by SA2 are presented in Figure 4. Exposure rates for the 11-year study period in SA2s where at least one exposure was notified (67 of 97 SA2s) ranged from 5 to 86 preventable potential ABLV exposures per 100000 people with a median of 2 (range 1 6) exposures per SA2. The highest exposure rates were in the northern SA2s of the region; Woodford - D'Aguilar, Wamuran, Upper Caboolture, Kilcoy and Dayboro. These are areas with low residential populations and large areas (all with less than 60 people per $\mathrm{km}^{2}$ ). In contrast, the highest numbers of preventable potential exposures were reported in different parts of Metro North HHS, including The Hills District SA3, in the west of the region with 21 exposures, and Caboolture SA3 and Narangba - Burpengary SA3 in the north of the region with 13 and 12 notifications respectively (Figure 5).

\section{Spatiotemporal clustering}

Two significant spatio-temporal clusters and one significant spatial cluster were identified in the Metro North HHS region (Figure 6, Table 3). Spatio-temporal Cluster $1(2012-2017)$ and Spatial 
Cluster 1 overlapped over 35 SA1s (12 notifications, area $785.5 \mathrm{~km}^{2}$, population 14835 ) although both clusters included areas and exposures unique to the cluster. These two clusters were located in the northern part of the region, and included regional towns such as Woodford, Dayboro and Upper Caboolture, and part of the Kilcoy, Woodford - D'Aguilar, Dayboro, Wamuran and Upper Caboolture SA2s. In addition to those mentioned above, Spatio-temporal Cluster 1 also included part of Elimbah, Caboolture, Caboolture - South and Morayfield and all of Wamuran and Upper Caboolture SA2s. No extra SA2s were included in Spatial Cluster 1. Six notifications in Spatial Cluster 1 were not included in Spatio-temporal Cluster 1 (two located outside the geographic area, four located within the geographic area but temporally outside the cluster). Seven notifications in Spatio-temporal Cluster 1 were geographically located outside Spatial Cluster 1 . Note that because clusters are identified based on circles encompassing SA1 centroids, areas with few or no cases may be included in the cluster if they are within a circle that increases the risk, such as the areas of Spatial Cluster 1 to the north and south of Spatio-temporal Cluster 1 included to capture two cases in the area to the west of Spatio-temporal Cluster 1.

Spatio-temporal Cluster 2 was a small cluster of less than five cases over a 2-year period (2012 2013), located in one SA1 in Redcliffe. Due to the small number of cases, the SA2 containing this SA1 is shown in Figure 6 to prevent reidentification.

In addition to the aforementioned significant clusters, there were seven non-significant spatial clusters (Table 2) and nine non-significant spatio-temporal clusters (Table 3). One spatio-temporal cluster (Cluster 5 in Table 3) included the whole of Metro North HHS for the period 2013 to 2017.

\section{Discussion}

Bat-human interactions occurred throughout the Metro North HHS region across the duration of study. While some interactions are not preventable (e.g. bat initiated or intentional handling by bat carers during the course of their work), intentional bat encounters by members of the public can be prevented. This study aimed to identify areas within the Metro North HHS region that might benefit from geographical targeting of ABLV prevention messages. During the 11-year period from 2007 to 2017, 144 preventable potential ABLV exposures in the general public were reported, an average annual rate of 1.4 exposures per 100000 population. This is lower than the average annual rate of 1.8 preventable potential ABLV exposures per 100000 population reported in the Metro South region during the twelve-year period from 1996 to 2008 (Megan K. Young \& McCall, 2010). People who reported preventable potential ABLV exposure were mostly adults and more likely to be male. The bats reported were most commonly megabats (e.g. flying foxes). The most commonly reported 
category of potential exposure was rescuing a trapped or injured bat $(71 \%)$. This is comparable to other studies including a study that found $84 \%$ of preventable exposures in NSW residents between 2007 and 2011 (Kardamanidis, Cashman, \& Durrheim, 2013) and 60\% of preventable exposures in QLD between 2009 and 2014 reported rescuing a trapped or injured bat (Si et al., 2016). A study on risk perception of the general public in NSW found that a combination of low awareness of the seriousness of $A B L V$ combined with a desire to help injured animals resulted in high numbers of people who said they would touch a bat and place themselves at risk of ABLV (Quinn et al., 2014).

In 2013, a child died of ABLV after exposure to a bat in Queensland (Francis et al., 2014). The media coverage around this event increased awareness of the risk of interactions with bats, which likely resulted in the increase in reported cases of potential ABLV exposure from 2013 (Figure 1). Indeed, the detection of a temporal cluster between 2013 and 2017 that incorporates the whole of Metro North HHS shows this increase.

Seasonality was evident in preventable potential ABLV exposures. The number of exposures reported increased during October and November, and again in January and February. Two of the three main megabat (flying fox) species present in Queensland give birth between September and November with the babies weaning in March (Wildcare Australia Inc, 2018). This corresponds with the increase in reported preventable potential ABLV exposures, likely due to increased activity of the bats leading to greater interaction with humans. It is unclear why reported exposures decrease during December. Increasing ABLV prevention messages around this time of year may reduce the incidence of preventable potential ABLV exposure in our region.

The highest rates of reported preventable potential ABLV exposures were in the sparsely populated SA2s in the north of Metro North HHS (Figure 4, Figure 5). Similarly, SaTScan analysis identified statistically significant clusters over both space and space-time in the same northern area of Metro North HHS (Figure 6), suggesting that this region would benefit from targeted public health messaging.

However, these are large areas with a relatively small number of potential exposures. While calculation of rates and cluster identification through SaTScan accounts for the underlying population, both methods use boundaries defined by the ABS to equalise populations in each region. Thus, SA1s and SA2s in the inner city are much smaller than those in rural areas of the HHS. Using a grid to define squares of equal area, regardless of the underlying population, identified a group of suburbs in the inner north of Brisbane with higher absolute numbers of exposures, suggesting this area would also benefit from targeted public health messaging. 
As with all studies, there are limitations to this study. Although we included all Metro North residents who were potentially exposed in Metro North and interviewed by staff of the Metro North Public Health Unit, it is likely there were additional people who were potentially exposed in Metro North but were interviewed at other public health units. The absence of these exposures from our analysis may have biased the results away from areas frequented by visitors to the region. In addition, reports of potential ABLV exposures are likely to be underreported, either deliberately by people not willing to risk sacrifice of the bat or due to a lack of knowledge of the potential seriousness of the disease and the need for prophylaxis.

The exact location of potential exposure was often not recorded on the CRF. As the true location for these exposures is unknown, it is possible that these exposures were allocated to the wrong geographic region (SA1, SA2, SA3, or grid square) on analysis, potentially biasing the geographic analysis. The effect of this bias is unknown.

While SaTScan is a robust methodology, it utilises centroids to represent geographic areas, rather than the polygon or the specific location of the exposure. We have minimised any errors this may introduce by using the smallest feasible ABS statistical area (SA1). However, SaTScan scans for clusters using a circle, despite clusters of disease rarely occurring in exact circles. These circles may include areas with few or no exposures.

Preventable potential ABLV exposures continue to occur in the Metro North HHS region which has an impact on public health resources. Seasonal analysis and spatiotemporal clustering of past events has highlighted the summer months and certain focus areas in the region where public health messages on bat handling and exposure to ABLV may be targeted in order to help alleviate future preventable potential exposure to ABLV.

\section{Conflict of interest statement}

The authors have no conflicts of interest to declare.

\section{References}

Australian Government Department of Health. (2013). Rabies virus and other lyssavirus (including Australian bat lyssavirus) exposures and infections CDNA National Guidelines for Public Health Units. Retrieved from http://www.health.gov.au/internet/main/publishing.nsf/Content/ED62D139B56F7B80CA25 7BF0001B7422/\$File/ABLV-Rabies-SoNG.pdf

Field, H. (2004). The Ecology of Hendra Virus and Australian Bat Lyssavirus. (PhD PhD Thesis), University of Queensland, Australia. 
Francis, J. R., Nourse, C., Vaska, V. L., Calvert, S., Northill, J. A., McCall, B., \& Mattke, A. C. (2014). Australian Bat Lyssavirus in a child: the first reported case. Pediatrics, 133(4), e1063-e1067. doi:10.1542/peds.2013-1782

G-NAF (CPSMA Australia Limited licensed by the Commonwealth of Australia under the Open Geocoded National Address File (G-NAF) End User Licence Agreement. (2018). Retrieved from: https://data.gov.au/dataset/geocoded-national-address-file-g-naf

Hanna, J. N., Carney, I. K., Smith, G. A., Tannenberg, A. E., Deverill, J. E., Botha, J. A., . . Searle, J. W. (2000). Australian bat lyssavirus infection: a second human case, with a long incubation period. Med J Aust, 172(12), 597-599.

Kardamanidis, K., Cashman, P., \& Durrheim, D. N. (2013). Travel and non-travel associated rabies post exposure treatment in New South Wales residents, Australia, 2007-2011: a crosssectional analysis. Travel Med Infect Dis, 11(6), 421-426. doi:10.1016/j.tmaid.2013.09.008

Kulldorff, M. (1997). A spatial scan statistic. Communications in Statistics - Theory and Methods, 26(6), 1481-1496. doi:10.1080/03610929708831995

McCall, B. J., Epstein, J. H., Neill, A. S., Heel, K., Field, H., Barrett, J., . . Lunt, R. (2000). Potential exposure to Australian bat lyssavirus, Queensland, 1996-1999. Emerg Infect Dis, 6(3), 259264. doi:10.3201/eid0603.000305

Merritt, T., Taylor, K., Cox-Witton, K., Field, H., Wingett, K., Mendez, D., ... Durrheim, D. (2018). Australian bat lyssavirus. Australian Journal for General Practitioners, 47, 93-96.

Murray, K., Morgan, J., \& Allworth, A. (1996). A human case of encephalitis due to a lyssavirus recently identified in fruit bats. Commun Dis Intell, 20(24), 504.

QGIS Development Team. (2018). QGIS Geographic Information System (Version Version 3.0.2): Open Souce Geospatial Foundation Project. Retrieved from http://qgis.osgeo.org

Public Health Act 2005, (2005).

Quinn, E. K., Massey, P. D., Cox-Witton, K., Paterson, B. J., Eastwood, K., \& Durrheim, D. N. (2014). Understanding human - bat interactions in NSW, Australia: improving risk communication for prevention of Australian bat lyssavirus. BMC Vet Res, 10, 144. doi:10.1186/1746-614810-144

Si, D., Marquess, J., Donnan, E., Harrower, B., McCall, B., Bennett, S., \& Lambert, S. (2016). Potential Exposures to Australian Bat Lyssavirus Notified in Queensland, Australia, 2009-2014. PLoS Negl Trop Dis, 10(12), e0005227. doi:10.1371/journal.pntd.0005227

Warrilow, D., Harrower, B., Smith, I. L., Field, H. E., Taylor, R., Walker, G. C., \& Smith, G. A. (2003). Public Health Surveillance for Australian bat lyssavirus in Queensland, Australia, 2000-2001. Emerging Infectious Disease journal, 9(2), 262. doi:10.3201/eid0902.020264

Wildcare Australia Inc. (2018). Bats. Retrieved from https://wildcare.org.au/speciesinformation/bats/

Wildlife Health Australia. (2017). ABLV Bat Stats. Australian Bat Lyssavirus Report - December 2017. Retrieved from

http://www.wildlifehealthaustralia.com.au/Portals/0/Documents/ProgramProjects/ABLV\%2 OBAT\%20STATS\%20Dec\%202017.pdf

Young, M. K., Banu, S., McCall, B. J., Vlack, S., Carroll, H., Bennett, S., . . Francis, D. (2018). Potential exposure to Australian bat lyssavirus is unlikely to prevent future bat handling among adults in South East Queensland. Zoonoses \& Public Health, 65(1), e237-e242. doi:10.1111/zph.12437

Young, M. K., El Saadi, D., \& McCall, B. J. (2014). Preventing Australian bat lyssavirus: community knowledge and risk perception of bats in South East Queensland. Vector Borne Zoonotic Dis, 14(4), 284-290. doi:10.1089/vbz.2013.1414

Young, M. K., \& McCall, B. J. (2004). Trends in potential exposure to Australian bat lyssavirus in South East Queensland, 1996 to 2003. Commun Dis Intell Q Rep(2), 258.

Young, M. K., \& McCall, B. J. (2010). Potential Exposure to Australian Bat Lyssavirus in South East Queensland: What Has Changed in 12 Years? Commun Dis Intell Q Rep(3), 334. 
Tables

Table 1 Descriptive statistics of preventable potential ABLV exposures in Metro North HHS ( $n=144)$

\begin{tabular}{|c|c|c|}
\hline \multicolumn{2}{|r|}{ Category } & n (\%) \\
\hline \multirow{2}{*}{ Age group } & Child (under 18 years) & $10(7)$ \\
\hline & Adult & $134(93)$ \\
\hline \multirow{2}{*}{ Sex } & Male & $87(60)$ \\
\hline & Female & $57(40)$ \\
\hline \multirow{5}{*}{ Exposure type } & Bite & $52(36)$ \\
\hline & Scratch & $74(51)$ \\
\hline & Bite and scratch & $10(7)$ \\
\hline & Mucous membrane & $2(1)$ \\
\hline & Unknown & $6(4)$ \\
\hline \multirow{3}{*}{ Bat type } & Megabat & $104(72)$ \\
\hline & Microbat & $24(17)$ \\
\hline & Unknown & $16(11)$ \\
\hline \multirow{4}{*}{ Category of contact } & Rescued a trapped or injured bat & $103(72)$ \\
\hline & Removed bat from animal or person & $26(18)$ \\
\hline & Removed bat from home & $13(9)$ \\
\hline & Other & $2(1)$ \\
\hline \multirow{3}{*}{ Exposure time } & Morning & $71(49)$ \\
\hline & Afternoon/evening & $69(48)$ \\
\hline & Unknown & $4(3)$ \\
\hline
\end{tabular}

Table 2 Spatial clusters of preventable potential ABLV exposures between 2007-2017 identified in Metro North HHS.

\begin{tabular}{|c|c|c|c|c|c|c|c|c|}
\hline Cluster & Number of SA1s & Area $\mathbf{( k m}^{\mathbf{2}} \mathbf{)}$ & Observed & Expected & Population $^{+}$ & RR & LLR & p-value \\
\hline 1 & 48 & 1714.51 & 18 & 3.21 & 20064 & 6.3 & 17.0 & 0.0001 \\
\hline 2 & 1 & 0.16 & 3 & 0.07 & 435 & 44.0 & 8.4 & 0.1829 \\
\hline 3 & 1 & 0.71 & 3 & 0.08 & 516 & 37.1 & 7.9 & 0.2759 \\
\hline 4 & 12 & 14.92 & 5 & 0.72 & 4491 & 7.2 & 5.5 & 0.9241 \\
\hline 5 & 1 & 2.01 & 2 & 0.05 & 320 & 39.7 & 5.4 & 0.9405 \\
\hline 6 & 1 & 0.17 & 2 & 0.06 & 358 & 35.4 & 5.2 & 0.9707 \\
\hline 7 & 4 & 6.57 & 3 & 0.22 & 1348 & 14.2 & 5.1 & 0.9749 \\
\hline 8 & 14 & 103.58 & 5 & 0.88 & 5503 & 5.9 & 4.6 & 0.9976 \\
\hline
\end{tabular}

RR: Relative risk; LLR:Log likelihood ratio

\footnotetext{
${ }^{+}$Averaged over time
} 
Table 3 Spatio-temporal clusters of preventable potential ABLV exposures between 2007-2017 identified in Metro North HHS.

\begin{tabular}{|c|c|c|c|c|c|c|c|c|c|}
\hline Cluster & $\begin{array}{c}\text { Number of } \\
\text { SA1s }\end{array}$ & Area $\left(\mathbf{k m}^{\mathbf{2}}\right)$ & Time period & Observed & Expected & Population & RR & LLR & $\begin{array}{c}\text { p- } \\
\text { value }\end{array}$ \\
\hline 1 & 89 & 841.82 & $2012-2017$ & 19 & 3.6 & 40045 & 5.9 & 17.1 & 0.001 \\
\hline 2 & 1 & 0.16 & $2012-2013$ & 3 & 0.01 & 435 & 236.6 & 13.4 & 0.04 \\
\hline 3 & 20 & 4.09 & 2013 & 4 & 0.11 & 7350 & 38.4 & 10.6 & 0.33 \\
\hline 4 & 1 & 0.71 & $2009-2013$ & 3 & 0.038 & 516 & 80.9 & 10.2 & 0.45 \\
\hline 5 & 2223 & 4268.08 & $2013-2017$ & 96 & 69 & 910167 & 2.1 & 10.0 & 0.51 \\
\hline 6 & 35 & 172.84 & $2012-2015$ & 7 & 0.82 & 14001 & 8.9 & 8.9 & 0.81 \\
\hline 7 & 2 & 1.85 & 2013 & 2 & 0.01 & 695 & 201.4 & 8.6 & 0.90 \\
\hline 8 & 1 & 0.47 & 2016 & 2 & 0.017 & 840 & 118.4 & 7.6 & 0.9952 \\
\hline 9 & 3 & 0.49 & 2007 & 2 & 0.017 & 1188 & 117.7 & 7.5 & 0.9956 \\
\hline 10 & 1 & 0.17 & $2010-2013$ & 2 & 0.021 & 358 & 95.8 & 7.1 & 0.9993 \\
\hline 11 & 2 & 1.53 & $2009-2010$ & 2 & 0.022 & 785 & 90.2 & 7.0 & 0.9995 \\
\hline
\end{tabular}

RR: Relative risk; LLR:Log likelihood ratio

${ }^{+}$Averaged over time

\section{Figure legends}

Figure 1. Number of preventable potential ABLV exposures in Metro North Hospital and Health Service, $2007-2017$

Figure 2. Mean number of preventable potential ABLV exposures per month January $2007-$ December 2018 (error bars represent standard deviation)

Figure 3. Heatmap grid over Metro North HHS showing number of notifications within each grid square. SA2 boundaries and some SA2 names are overlaid for orientation.

Figure 4. Notification rates of preventable potential ABLV exposures in Metro North HHS 2007-2017, by SA2

Figure 5. Notifications of preventable potential ABLV exposures in Metro North HHS 2007-2017 by SA3

Figure 6. Clusters of higher than expected numbers of preventable potential ABLV exposures over space alone or space and time, Metro North HHS 2007-2018, with SA1 clusters coloured for Spatiotemporal Cluster 1 and Spatial Cluster 1. The SA2 containing Spatio-temporal Cluster 2 is coloured as this cluster has less than 5 cases in a small area. Spatio-temporal Cluster 1 was significant between 2012-2017 ( $p=0.001$ ), Spatio-temporal Cluster 2 was significant 2012-2013 ( $p=0.04)$, and Spatial Cluster 1 was significant $(p<0.001)$. Temporal cluster 1 and Spatial Cluster 1 partially overlap (shown in darker purple). 